\title{
SPECTROSCOPIC CCD SEARCH FOR THE UNSEEN COMPANIONS IN THREE SINGLE-LINE WNL BINARIES
}

\author{
ANDRE GRANDCHAMPS and ANTHONY F. J. MOFFAT \\ Département de physique, Université de Montréal, Montréal \\ and Observatoire du mont - Mégantic
}

We have obtained some 15 CCD spectra at different orbital phases for each of the SB1 systems HD193928 (WN6), HD197406 (WN7), and CQ Cep (WN7), in an attempt to detect the companion, determine its orbit and estimate the masses. The wavelength range of the spectra is $\lambda \lambda 3600-4200 \AA$, with $1.4 \AA /$ pixel and $\langle S / N\rangle \approx 100 /$ pixel. We compare the best emission-line (N IV $\lambda 4058 \AA$ ) with the best absorption-line (H9 $\lambda 3835 \AA$ ).

For HD193928 we find $K_{W N G}=128 \mathrm{~km} \mathrm{~s}^{-1}$ and $K_{O}=133 \mathrm{~km} \mathrm{~s}^{-1}$. With $i=71.7^{\circ}$, based on polarisation data (Grandchamps et al., 1990. in preparation), we obtain $M_{W N G}=23.8 \pm 8.6 M_{\odot}$ and $M_{\odot}=22.9 \pm 3.3 M_{\odot}$.

For HD197406 the WN7 star appears to be too luminous to allow a detection of its companion with the present technique.

For CQ Cep the absorption-line follows the same motion as the emission-line and thus arises in the WR star itself.
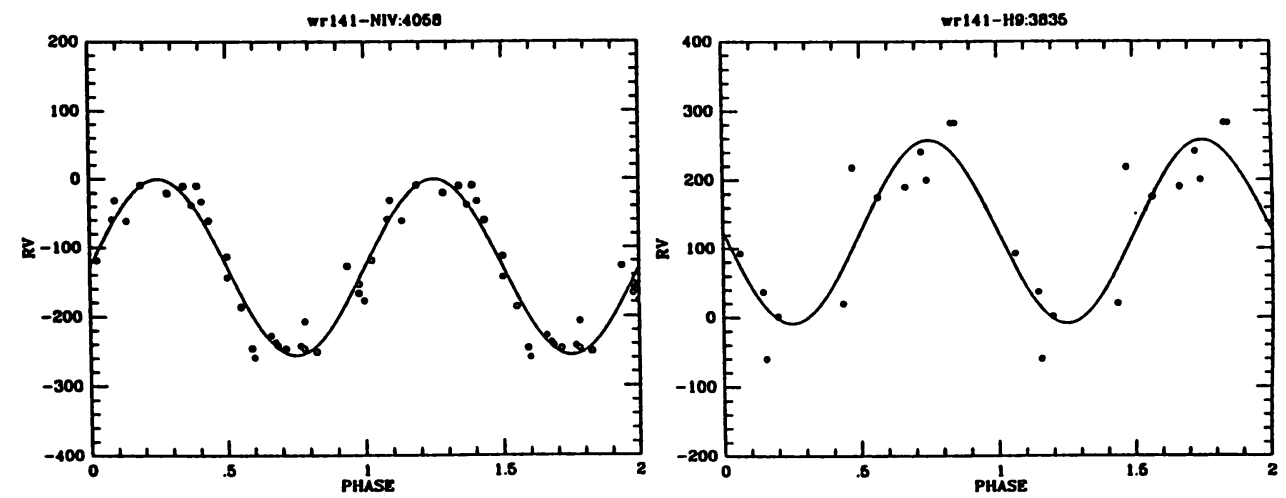

Fig. 1: Velocity sine curve (radial velocities in $\mathrm{km} \mathrm{s}^{-1}$ ) fit to the data of HD193928. The left half refers to N IV $\lambda 4058 \AA$ emission-line; the right half to H9 $\lambda 3835 \AA$ absorptionline. The open circles representent the Ganesh and Bappu (1968, Kodaikanal Observatory Bulletin Ser. A. 185, A104) data; filled circles our data.

K. A. van der Hucht and B. Hidayat (eds.),

Wolf-Rayet Stars and Interrelations with Other Massive Stars in Galaxies, 258.

(c) 1991 IAU. Printed in the Netherlands. 